\title{
LncRNA RPL13p5 gene expression promotes insulin resistance in patients with gestational diabetes
}

\author{
Yuanyuan $\mathrm{Li}^{1,2} \wedge$, Xingbo Cheng ${ }^{1 \#}$, Dongmei $\mathrm{Li}^{2 *}$ \\ ${ }^{1}$ Department of Endocrinology, The First Affiliated Hospital of Soochow University, Suzhou, China; ${ }^{2}$ Department of Endocrinology, Inner Mongolia \\ Autonomous Region People's Hospital, Hohhot, China \\ Contributions: (I) Conception and design: Y Li, X Cheng; (II) Administrative support: X Cheng, D Li; (III) Provision of study materials or patients: \\ D Li, Y Li; (IV) Financial support: D Li; (V) Collection and assembly of data: Y Li; (VI) Data analysis and interpretation: Y Li; (VII) Manuscript \\ writing: All authors; (VIII) Final approval of manuscript: All authors. \\ \#These authors contributed equally to this work. \\ Correspondence to: Xingbo Cheng. Department of Endocrinology, The First Affiliated Hospital of Soochow University, Suzhou 215000, China. \\ Email: cxbszdxfsdyyy@163.com; Dongmei Li. Department of Endocrinology, Inner Mongolia Autonomous Region People’s Hospital, 20 Zhaowuda \\ Street, Hohhot 010000, China. Email: 1dm1229@126.com.
}

\begin{abstract}
Background Gestational diabetes mellitus (GDM) is a universal disease caused by a combination of environmental and genetic factors and resulting in abnormal signaling pathway function and eventual islet cell insufficiency. According to co-expression profile network data between messenger RNA (mRNA)-long non-coding RNA (lncRNA) in our laboratory, we selected lncRNA RPL13P5 as a candidate lncRNA related to the GDM insulin signaling pathway and which is upregulated in GDM patients.
\end{abstract}

Methods: The study included a gestational diabetes group $(n=25)$ and normal pregnancy group $(n=19)$. Expression was detected by quantitative polymerase chain reaction (qPCR) and showed the expression levels of lncRNA RPL13P5 in peripheral blood were significantly different between the two groups $(\mathrm{P}<0.05)$. The correlation between the homeostasis model assessment insulin resistance (HOMA-IR) and lncRNA expression was analyzed.

Results: The lncRNA RPL13p5 forms a co-expression strand with the TSC2 gene via the PI3K-Akt signaling pathway. The sequencing of lncRNA RPL13P5 showed high expression and the qPCR validation results were consistent with the sequencing results. An association was observed between the expression level of lncRNA RPL13P5 and other clinicopathological features of GDM.

Conclusions: LncRNA RPL13P5 forms a co-expression chain with the TSC2 gene through the PI3K-Akt signaling pathway and becomes part of the process of insulin resistance in GDM.

Keywords: Gestational diabetes; long non-coding RNA (lncRNA); lncRNA RPL13p5; insulin signaling pathway

Submitted Aug 05, 2021. Accepted for publication Oct 22, 2021.

doi: 10.21037/apm-21-2940

View this article at: https://dx.doi.org/10.21037/apm-21-2940

\section{Introduction}

Gestational diabetes mellitus (GDM) is defined as diagnosed diabetes mellitus during gravidity in women with normal glucose metabolism or with underlying hypoglycemia before pregnancy. The worldwide prevalence is $1 \%$ to $14 \%$, with a significant increase in recent years (1). GDM is a common complication of pregnancy and has a major impact on the growth and development of the children of affected mothers, while the likelihood of the latter developing type 2 diabetes in the future is much higher than in the general

$\wedge$ ORCID: 0000-0002-7923-8251. 
population (2). In mid to late pregnancy, the level of antagonistic insulin substances increases, causing sensitivity to insulin to decrease and making insulin secretion relatively insufficient (3). For pregnant women with restricted insulin secretion, gestation cannot compensate for this physiological change and elevated blood glucose and decreased $\beta$-cell function result from insulin resistance. The pathogenesis of GDM has not been fully elucidated and involves genetic factors, environmental factors (4), autoimmunity, placental hormones, and inflammatory mediators. However, which factors, and through which pathways they act to cause excessive insulin resistance in patients with GDM, requires further investigation. With the in-depth study of gene regulatory networks, research has gradually focused on exploring the molecular level of GDM, and has found that the abnormal expression of insulin resistance-related genes gradually accumulates, leading to abnormal function of related signaling pathways and eventually defective islet cell function. Due to the lack of typical clinical symptoms in GDM patients, conventional diabetes mellitus examination cannot accurately determine early insulin resistance in a timely manner, and a molecular marker is needed to detect this. In recent times, the relationship between long non-coding RNAs (lncRNAs) and diabetes has received increasing attention (5), but the relationship between lncRNA and GDM expression is less well studied.

DNA is used as a template for genetic information in the nucleus to guide the formation of RNA for the synthesis of proteins by transcription and translation. The human genome is currently known to contain about 25,000 genes. In the organic synthesis of DNA templates, most non-coding RNA (ncRNA) are not transcribed and only $2 \%$ of the genome is expressed as proteins (6). Among them, lncRNAs, which are widely present in eukaryotic cells, are involved in regulating the rate and process of genetic information and the synthesis and breakdown of many nutrients (7), as well as playing a positive role in normal physiological activities (8-10). LncRNA expression is stable, highly specific, and not easily destroyed, and is stably expressed in blood and urine. In recent years, with the development and widespread appositeness of high-throughput RNA-sequencing (RNAseq), lncRNAs have become a new target of interest in ncRNAs, including their role in diabetes and insulin resistance (10-12). However, we found few studies examining the association of insulin resistance and lncRNA by highthroughput based on patients with gestational diabetes, such as microarrays and RNA-seq. In our previous application (13), which reported the expression profile of gestational diabetes
IncRNA. In this study, the transcriptome sequences were compared with public databases to derive differential expression of lncRNAs and messenger RNAs (mRNAs). LncRNA microarray analysis identified 7,498 genes (3,592 up-regulated and 3,906 down-regulated) differentially expressed in the GDM group and healthy pregnant controls, including 1,098 differentially expressed lncRNAs (609 upregulated and 489 down-regulated) (Tables 1,2, Figure 1). By biofunctional clustering and KEGG signaling pathway analysis, we selected mRNAs and lncRNAs that are closely related to the insulin signaling pathway in GDM and analyzed their possible regulatory mechanisms in the pathogenesis of GDM. Most differential lncRNAs being associated with islet differentiation effects. The sequencing results were further validated by quantitative polymerase chain reaction (qPCR). Based on the regulatory pathways of the lncRNA mRNA network, their co-expression network was constructed by Pearson correlation analysis (Figure 2). The degree function represents the degree of interrelationship between a node and other nodes in a virtual network and the number of nodes in the network. The lncRNAs ERMP1, TSPAN32 and MRPL38 were found to form a co-expression network with TPH1, which is mainly involved in the tryptophan metabolic pathway and in the development of GDM. In addition, lncRNA RPL13P5 formed a co-expression network with TSC2 gene through PI3K-Akt and insulin signaling pathway and was involved in the process of insulin resistance in GDM.

There was no significant difference in the expression of PTH in the qPCR validation of the GDM group $(\mathrm{n}=3)$ vs. the control group ( $n=3)$, which was not consistent with the transcriptional sequencing results. TSC2 was upregulated in the peripheral blood of GDM patients, and the qPCR validation results were consistent with the sequencing results. The sequencing of lncRNA RPL13P5, which forms a co-expression pair with TSC2, showed high expression, and the $\mathrm{qPCR}$ validation results were consistent with the sequencing results. The co-expressed mRNA-lncRNA: lncRNA-RPL13P5-TSC2 was selected for quantitative reverse transcription PCR (qRT-PCR) validation (Table 3). We then designed primers to verify if differential expression was present (Table 4). indicating whether expression was significantly higher in GDM than in normal pregnant women $(\mathrm{P}<0.0041 ; t=5.908)$ (Figure 3$)$. Our studies have shown the gene encoding lncRNA RPL13P5 was stably and distinguishingly expressed in the outside blood of GDM in preliminary experiments, resulting in us proposing RPL13P5 could be a novel molecular marker of GDM and a potential molecular marker for its early diagnosis. In a mechanistic 
Table 1 Top 10 differentially expressed lncRNAs

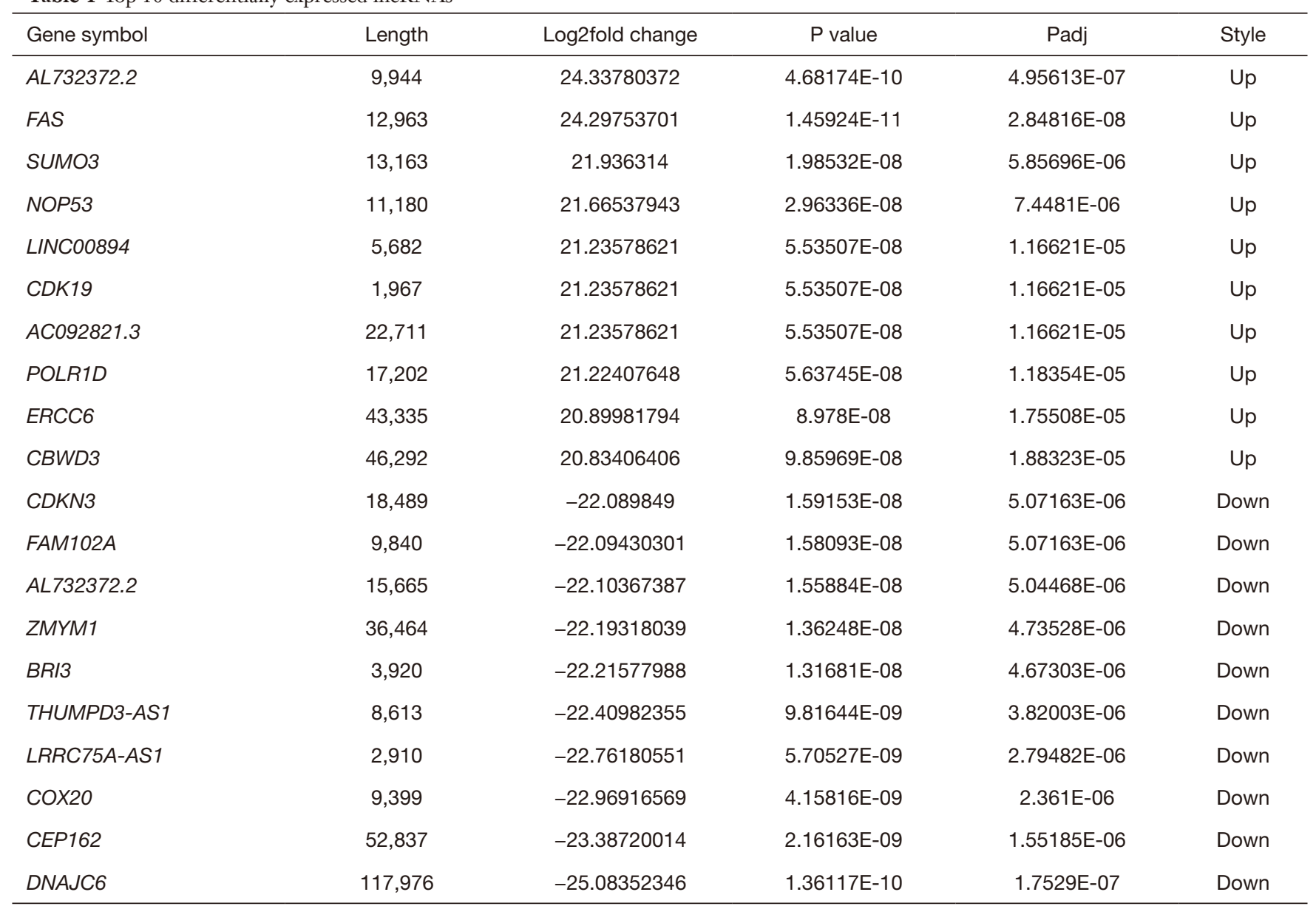

LncRNA, long non-coding RNA.

study, we also combined the analysis of recent studies, and showed RPL13P5 may be involved in multiple key signaling pathways in the development of insulin resistance (1). We then verified that its differential expression was consistent with our previous findings based on the unique structure of lncRNA, which was conserved, stable, and tissue-specific, indicating it could be regarded as an early indicator for the diagnosis and prognosis assessment of GDM.

We present the following article in accordance with the MDAR reporting checklist (available at https://dx.doi. org/10.21037/apm-21-2940).

\section{Methods}

\section{Data collection}

The flow diagram for this study is presented in Figure 4. We selected randomly 25 patients with GDM and 19 normal pregnant women newly diagnosed in our obstetrics outpatient clinic from October 2019 to March 2020. The study was approved by the Health Office Ethics Commission of the Inner Mongolia Autonomous Region People's Hospital (201811005) and all study subjects signed inform consent documentation. All procedures performed in this study involving human participants were in accordance with the Declaration of Helsinki (as revised in 2013). The body mass index (BMI) of patients at 36 weeks gestation, as well as fasting blood glucose (FBG), 1-hour glucose load, 2-hour glucose load, fasting serum insulin, and c-peptide levels at 24-28 weeks were extracted, and based on the collected information, we calculated a homeostasis model assessment insulin resistance (HOMA-IR) to assess the insulin resistance status in both groups.

Calculation formula: HOMA-IR = fasting insulin (FINS) $\times \mathrm{FBG} / 22.5$, as described in the reference (14). 
Table 2 Top 10 differentially expressed mRNAs

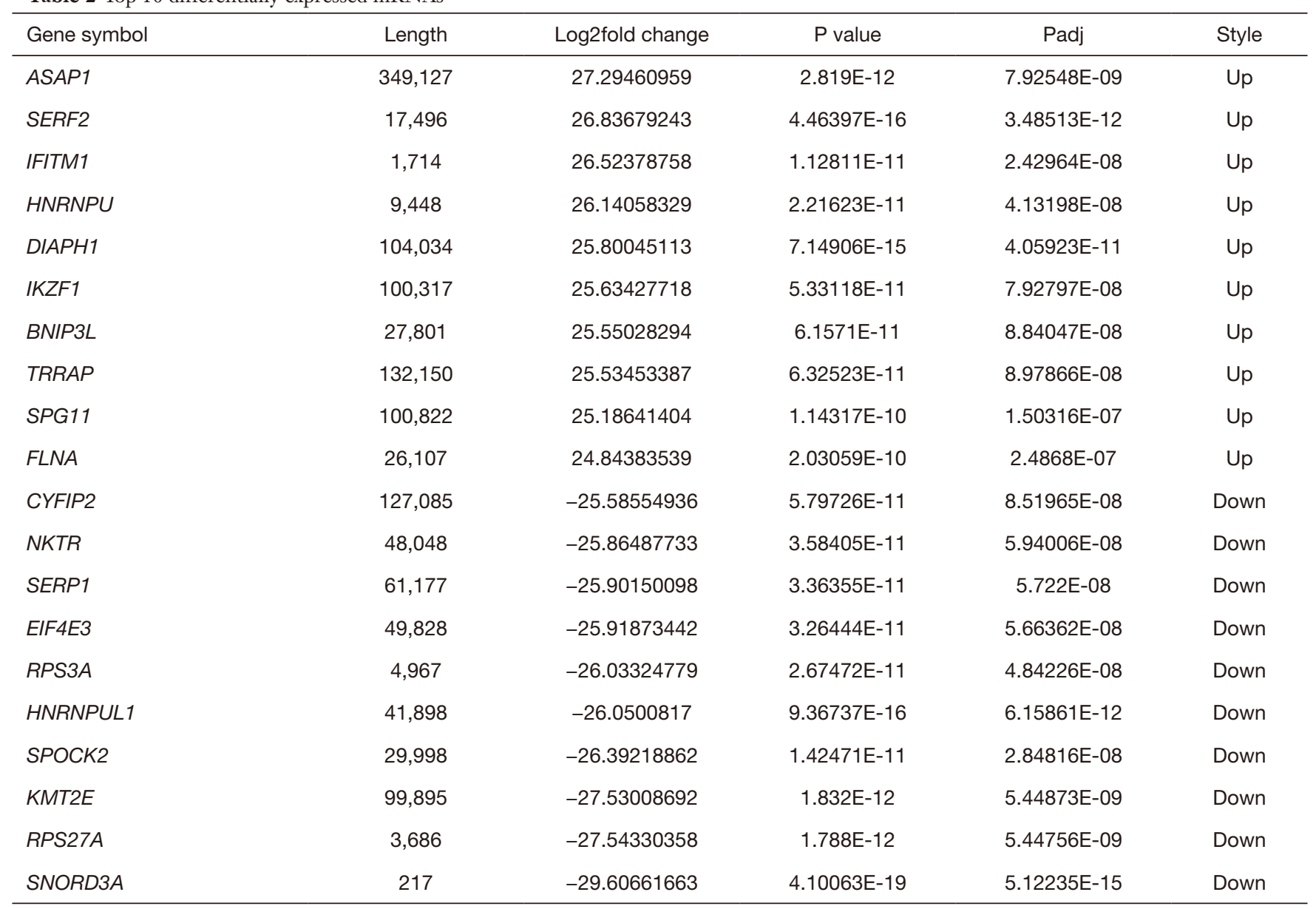

mRNAs, messenger RNA.

The diagnosis of GDM relied on the American Diabetes Association (ADA) 2019 standard (15) and was considered present when oral glucose tolerance test (OGTT) measurement for the glycemic load test showed blood glucose level exceeded any of the specified cutoff values [fasting: $92 \mathrm{mg} / \mathrm{dL}(5.1 \mathrm{mmol} / \mathrm{L}) ; 1 \mathrm{h:} 180 \mathrm{mg} / \mathrm{dL}(10.0 \mathrm{mmol} / \mathrm{L})$; $2 \mathrm{~h}: 153 \mathrm{mg} / \mathrm{dL}(8.5 \mathrm{mmol} / \mathrm{L})]$. As previously stated (15), those with pre-pregnancy diabetes, high blood pressure, pre-eclampsia, or obesity (BMI) $\geq 30$ (16) were excluded from the trial (13).

\section{Maternal characteristics}

There was no significant difference in the age and BMI between the two groups. In contrast, fasting plasma glucose (FPG), OGTT-1 h blood glucose, OGTT-2 h blood glucose, insulin (mU/L), and HOMA-IR were checked at 36 weeks of pregnancy, and the differences between groups was significant. A sample of this data is shown in Table 5 . The original data sources can be found in the published article on the study (13).

\section{Extraction and qPCR}

According to the co-expression profile network data between mRNA-lncRNA in our laboratory, we selected RPL13P5 as a candidate IncRNA related to the GDM insulin signaling pathway. Expanded samples were validated in the experimental group with GDM (Exp $=25$ ) and control group of healthy pregnant women (Con =19). Primer sequences for qPCR of lncRNARPL13P5 are that RPL13P5-F: GGGCTTAACCCTCTCATCG, 
A

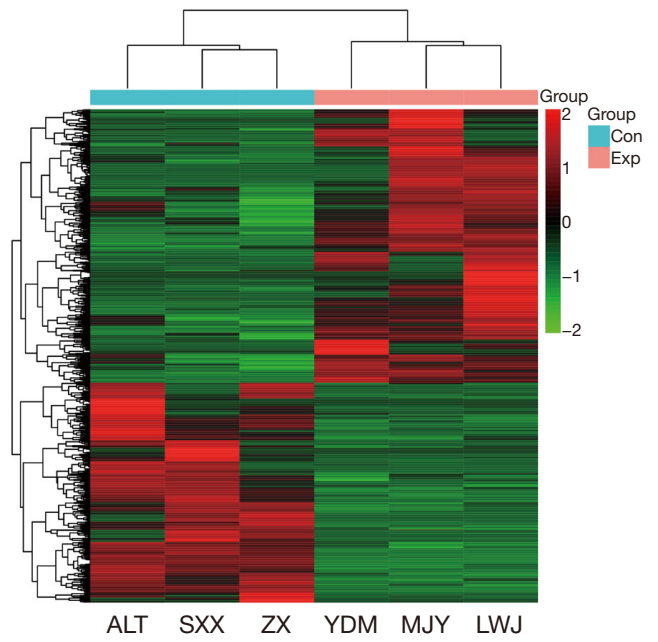

C

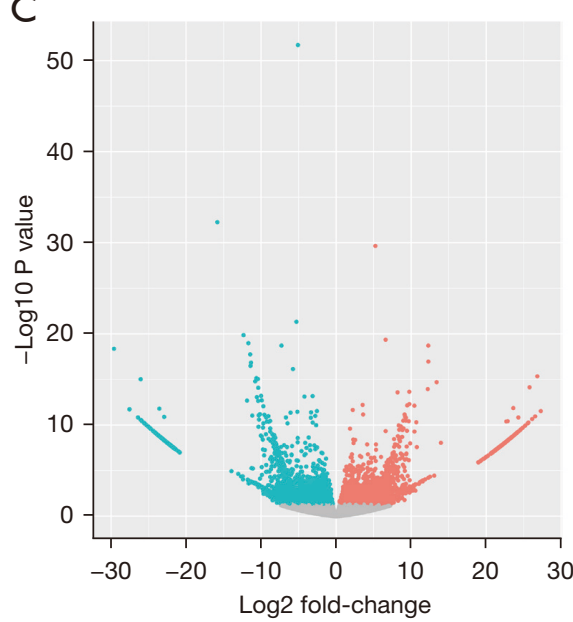

B

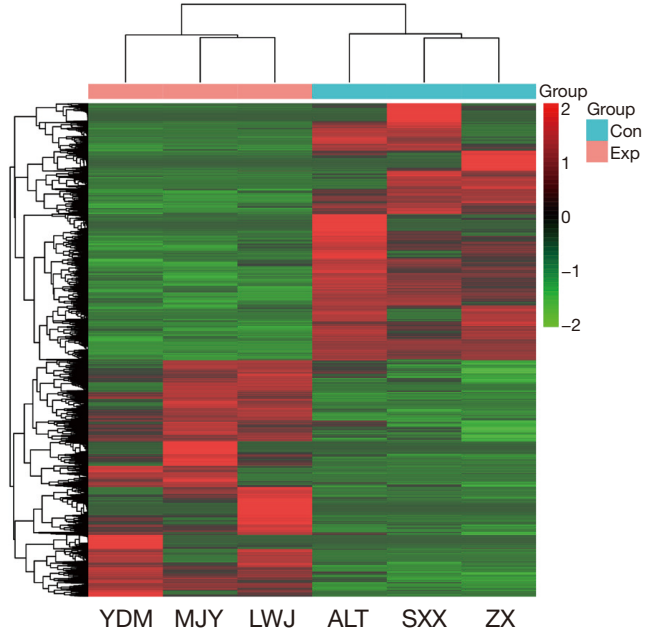

D

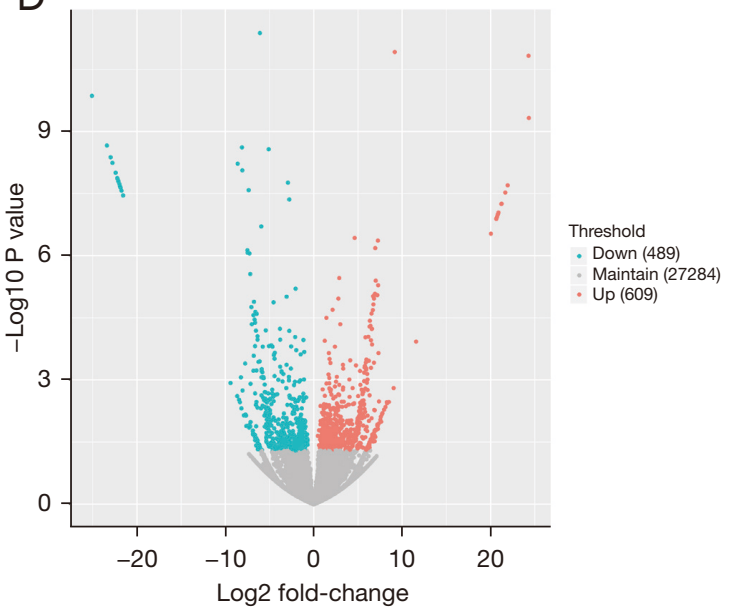

Figure 1 Heat map and volcanic analysis for the differentiated expressing lncRNAs and mRNAs. (A) Heatmap analyses of differentially expressed mRNAs. (B) Heatmap analyses of differentially expressed lncRNAs. (C) Volcano analyses of differentially expressed mRNAs. (D) Volcano analyses of differentially expressed lncRNAs. LncRNA, long non-coding RNA; mRNA, messenger RNA.

RPL13 P 5-R: GGGACTGAGACCCGAGGTTA. ChamQ ${ }^{\mathrm{TM}}$ SYBR qPCR Master Mix (vazyme Q311-02) was used to perform real-time fluorescent qPCR amplification experiments to verify the sequencing results (Table 4). Monitoring was also performed using the Gene Amp PCR System 9700 .

\section{Correlation analysis between IncRNA level and insulin resistance}

Insulin resistance was indirectly evaluated using the homeostatic index of insulin resistance. The correlation between the HOMA-IR and lncRNA expression was analyzed using Spearman correlation analysis.

\section{Statistical analysis of lncRNA}

Two independent samples were tested using $t$-test to make comparisons of differential gene expression levels in peripheral blood of both groups using SPSS22.0 statistical software with a $\mathrm{P}$ value of less than 0.05 used as the standard for calculation. Multiple linear regression was used for analysis of the relationship of lncRNA RPL13P5 to insulin resistance in GDM. 


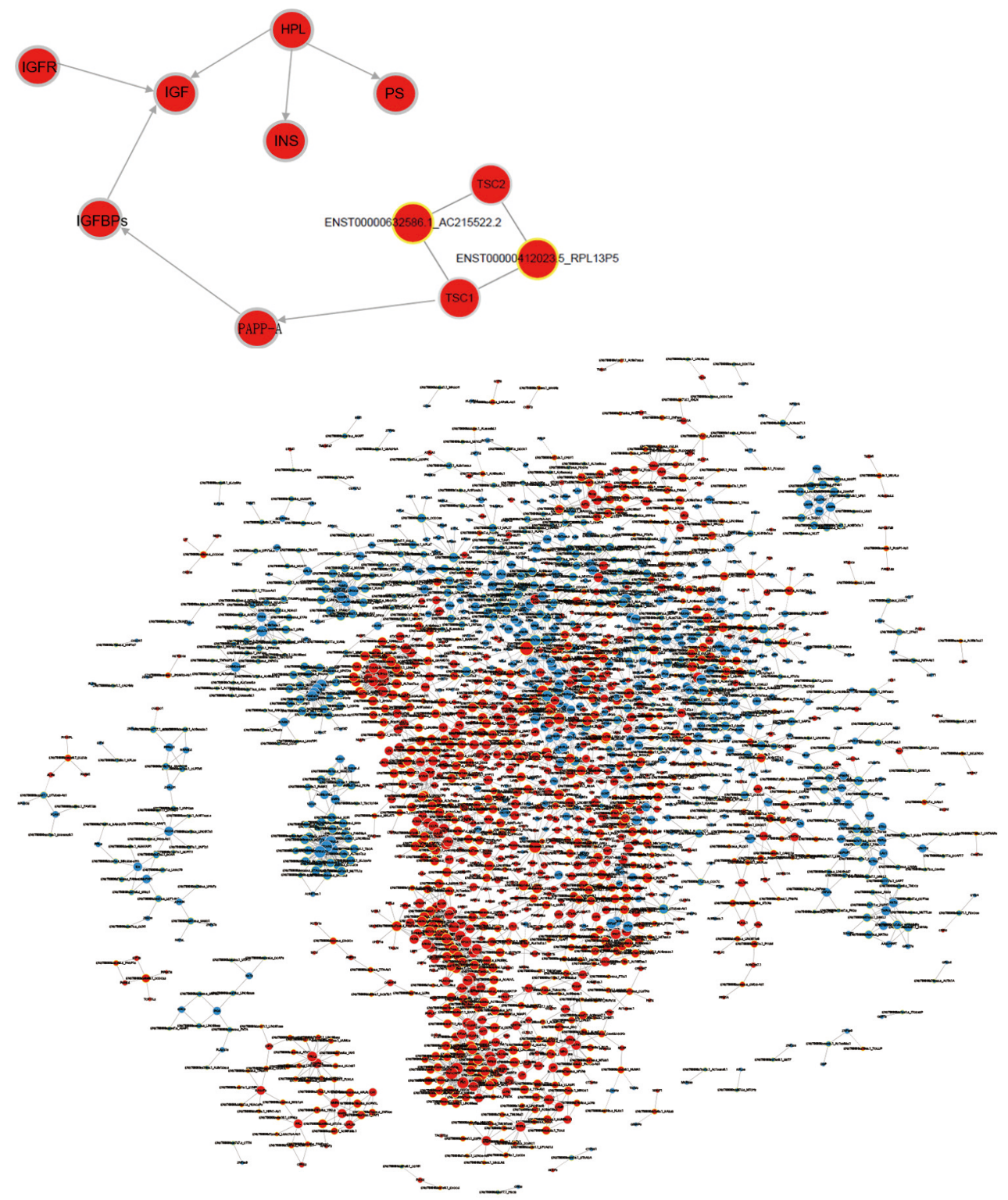

Figure 2 mRNA-lncRNA co-expression network. mRNA, messenger RNA; lncRNA, long non-coding RNA.

Table 3 Partial results of differential genes and lncRNA attributes were presented

\begin{tabular}{lcccc}
\hline Gene symbol & Degree & Log2fold change & Biotype & Gene feature \\
\hline ENST00000412023.5_RPL13P5 & 7 & 1.069003946 & Up & Noncoding \\
TSC1 & 1 & 1.848237074 & Up & Coding \\
\hline
\end{tabular}

LncRNA, long non-coding RNA. 
Table 4 Primers for qPCR of lncRNA RPL13P5

\begin{tabular}{ll}
\hline Amplification primer name & Amplification primer sequence \\
\hline RPL13P5-F & GGGCTTAACCCTCTCATCG \\
RPL13P5-R & GGGACTGAGACCCGAGGTTA \\
TSC1-F & CTCCACAGCCAGATCAGACA \\
TSC1-R & ACACCTTGTTGTTGGCCTTC \\
TSC2-F & AGTGGTCATCTCGCAGCTCT \\
TSC2-R & GCCATCACCTTCTCGATGAT
\end{tabular}

qPCR, quantitative polymerase chain reaction; IncRNA, long non-coding RNA.

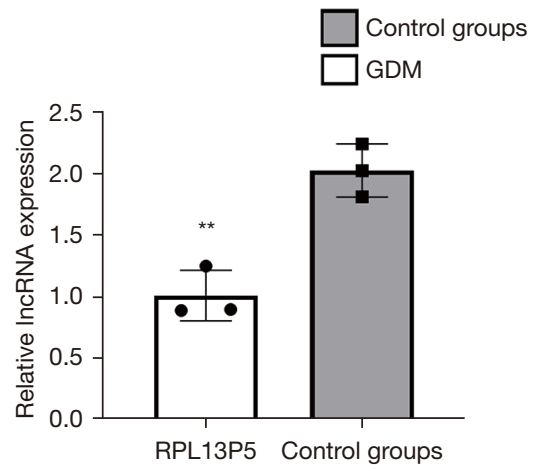

Figure 3 The GDM group (n=3) vs. the control group (n=3) qPCR to validate the expression of lncRNA RPL13P5. **, $\mathrm{P}<0.01$. GDM, gestational diabetes mellitus; qPCR, quantitative polymerase chain reaction; lncRNA, long non-coding RNA.

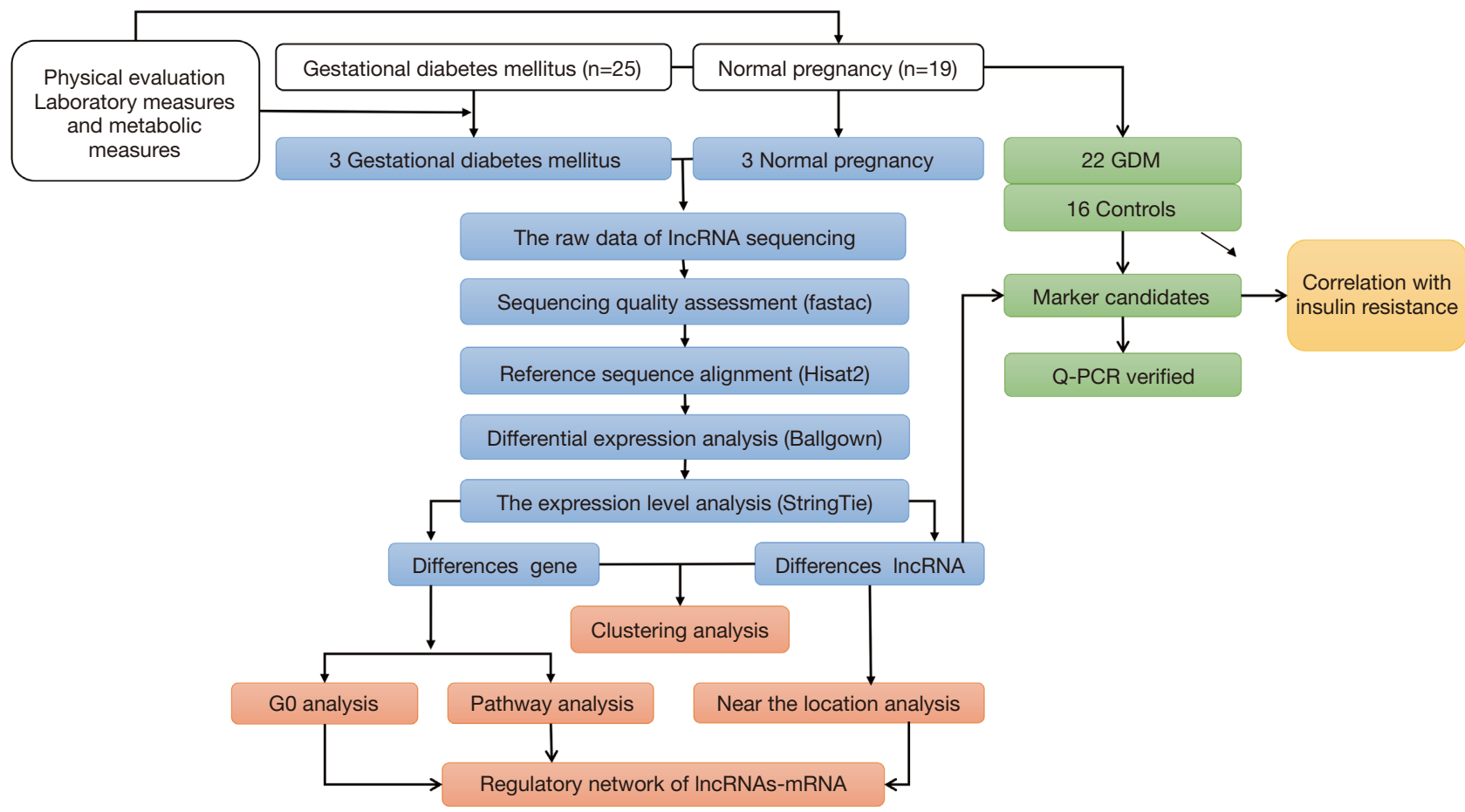

Figure 4 Flow diagram for this study. GDM, gestational diabetes mellitus; lncRNA, long non-coding RNA; qPCR, quantitative polymerase chain reaction; mRNA, messenger RNA.

\section{Results}

\section{Validation of IncRNA RPL13P5 expression pattern by qPCR}

According to the mRNA lncRNA differential expression profile network, lncRNA RPL13P5 related to the GDM insulin signaling pathway was selected for qPCR verification. We designed primers to verify the differential expression from the control group to the experimental group (Table 4).

The expression level of lncRNA RPL13P5 was significantly different between the two groups, and its expression in the external blood of GDM was highly statistically significant 
Table 5 Characteristics of study participants

\begin{tabular}{lccc}
\hline Characteristics & GDM $(\mathrm{n}=25)$ & Control group $(\mathrm{n}=19)$ & $\mathrm{P}$ value \\
\hline Age, y & $31.68 \pm 0.7499$ & $30.16 \pm 0.8661$ & 0.1907 \\
Pre-pregnancy BMI & $23.79 \pm 0.6881$ & $21.49 \pm 0.7277$ & 0.0287 \\
FBG (mmol/L) & $5.739 \pm 0.3522$ & $4.286 \pm 0.0531$ & 0.0009 \\
OGTT-1 h & $10.82 \pm 0.7044$ & $7.154 \pm 0.3189$ & 0.0001 \\
OGTT-2 h & $9.264 \pm 0.4022$ & $6.079 \pm 0.2557$ & $<0.0001$ \\
Insulin (mU/L) & $11.41 \pm 1.129$ & $6.552 \pm 0.5757$ & 0.0012 \\
HOMA-IR & $2.62 \pm 0.2714$ & $1.246 \pm 0.1083$ & 0.0001 \\
\hline
\end{tabular}

GDM, gestational diabetes mellitus; BMI, body mass index; FBG, fasting blood glucose; OGTT, oral glucose tolerance test; HOMA-IR, homeostasis model assessment insulin resistance.
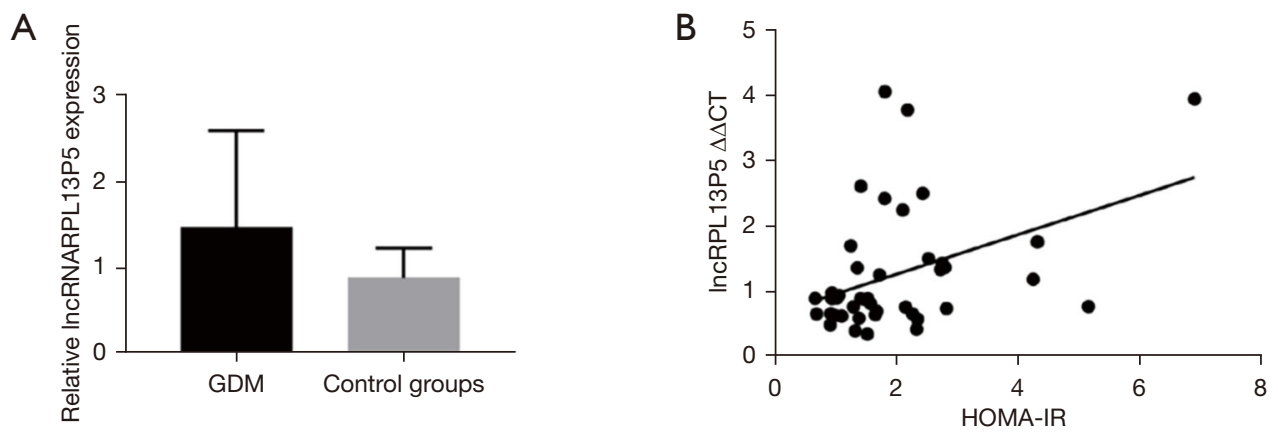

Figure 5 Validation of lncRNA RPL13P5 expression pattern by qPCR and association between the expression level of lncRNA RPL13P5 and other clinicopathological features of GDM. (A) qPCR of lncRNARPL13P5 expression levels in peripheral blood of patients with GDM. (B) Correlation analysis between lncRNA level and insulin resistance. LncRNA, long non-coding RNA; qPCR, quantitative polymerase chain reaction; GDM, gestational diabetes mellitus.

in the experimental group compared with the control group $(\mathrm{P}<0.05)$ (Figure $5 A)$.

An association was observed between the expression level of lncRNA RPL13P5 and other clinicopathological features of GDM (Table 6, Figure 5B), indicating it might have a biological function within the metabolic process of GDM, and deserves further investigation.

\section{Discussion}

Excessive insulin resistance and potential impairment of pancreatic $\beta$-cell function during pregnancy are the pathophysiological basis of GDM, and which genes participate in insulin resistance and are involved in the process of pancreatic $\beta$-cells is worthy of investigation. Human placental prolactin (HPL) is secreted in high levels during pregnancy and has a variety of biological functions, including lactation regulation, morphogenesis, reproduction, metabolism and adaptation to physiological stressors. HPL can accelerate lipolysis and inhibit the deposition of fat in the body, thereby increasing the level of free fatty acids in the blood, which are used as an energy source to maintain physiological functions. Insulin in the surrounding tissues is inhibited, the muscular uptake of glucose is reduced, the utilization rate of blood glucose is greatly reduced, and the increase in blood glucose is conducive to the ingestion of a large amount of glucose from the mother's blood by the fetus for growth and development. HPL regulates glucose metabolism through insulin signal-related pathways and participates in regulating the steady-state adaptation of glucose to pregnancy (17). Therefore, the function of HPL is an important condition for the rapid growth and development of the fetus. Studies have shown that patients with GDM 
Table 6 Linear regression analysis with GDM HOMA-IR as the dependent variable

\begin{tabular}{|c|c|c|c|c|c|}
\hline Model & Non-standardized coefficient B & Standard error & Standard coefficient & $t$ & Sig. \\
\hline BMI & 0.111 & 0.075 & 0.012 & 1.479 & 0.16 \\
\hline FPG & -0.501 & 0.271 & -0.028 & -1.847 & 0.085 \\
\hline FINS & 0.622 & 0.016 & 1.089 & 39.925 & 0 \\
\hline OGTT-1 h & 0.057 & 0.124 & 0.006 & 0.46 & 0.652 \\
\hline OGTT-2 h & -0.092 & 0.096 & -0.013 & -0.965 & 0.35 \\
\hline LncRNA RPL13P5 & -0.495 & 0.197 & -0.015 & -2.511 & 0.024 \\
\hline
\end{tabular}

A dependent variable: insulin resistance index. GDM, gestational diabetes mellitus; HOMA-IR, homeostasis model assessment insulin resistance; BMI, body mass index; FPG, fasting plasma glucose; FINS, fasting insulin; OGTT, oral glucose tolerance test; IncRNA, long noncoding RNA.

have excessive insulin resistance characterized by a fifty percent decline in insulin susceptibility in humans with the doubling of insulin secreted. Insulin resistance is one of the early determinants of declining cellular function (18), and following a threshold, inadequate insulin production and increasing insulin requirements will result in hyperglycemia in patients with GDM (19). Patients with GDM have increased insulin-like growth factor (IGF) (20), hormone insulin, adrenocorticotropic hormone, as well as pulmonary surfactant (PS), which enhances fetal access to glucose and essential amino acids, resulting in a large fetal weight at delivery.

In our analysis of the co-expression network, the upregulation of lncRNA RPL13P5 is correlated with the expression of STC2, and to further study the biological function of lncRNA RPL13P5, we chose stann-calcin-2 (STC2) for targeted genetic analysis. TSC is composed of the genes TSC1 and TSC2, which are widely expressed in various tissues of the body, encoding hamartin and tuberin proteins separately.

The TSC1/TSC2 gene is in the center of the signal transduction pathway and is mainly enriched in the mTOR signal pathway, endocrine and metabolic-related signal pathways such as PI3K/AKT, insulin signal pathway, insulin resistance, and other signal pathways (21). Overexpression of $S T C 2$ increased the activity of PI3K-p $85 \alpha$ and phosphorylated AKT (Ser473). Therefore, we speculated that the significant overexpression of IncRNA RPL13P5 in GDM patients may mainly promote insulin resistance through PI3K/Akt signaling.

We also found that STC2 effectively inhibited proteinolytic activity of pregnancy-associated plasma protein-A (PAPP-A). Cys-120 and PAPP-A of STC2 are covalently bound by disulfide bonds to reduce proteolysis, and PAPP-A is a protein catabolic enzyme. Studies have found (22) that on the one hand, PAPP-A can activate insulin-like growth factor receptor (IGFR) by dissolving lecithin, and on the other hand, it can hydrolyze insulin-like growth factor binding protein (IGFBPs) such as IGFBP-4. IGFBP-4 competes with IGFR for binding to IGFs (23), and IGFs bound to IGFBP-4 are unable to exert biological activity. IGFBP-4 protein splits and decomposes to release biologically active IGF, which is activated by PAPP-A, thereby initiating IGF signals and ultimately regulating its biological functions. The effect of enhancing local IGF has been confirmed in many studies both in vivo and in ex vivo (24). The association with STC2 as well as PAPP-A prevents the cleavage of IGFBP-4. In line with this, STC2 proved to be effective in inhibiting in vitro PAPP-A regulated IGF acceptor signaling (25). The upregulation of lncRNA RPL13P5 is related to the expression of STC2. As an RNA that is stabilized and measurable, lncRNA RPL13P5 may be used to cure disorders through complementary down-regulation of ncRNA expression and inhibition of ncRNA overexpression. This shows lncRNA $R P L 13 P 5$ is a potential biomarker for GDM.

The lncRNA transcripts associated with the GDM insulin signaling pathway are mainly enriched in both the PI3K/AKT signal pathway and the insulin signature pathway. IncRNA RPL13P5 is involved in the process of insulin resistance in GDM by forming a co-expression network by the PI3K-Akt signaling pathway, insulin signal 
pathway, and TSC2 gene. Currently, most research on lncRNA is on diabetes mellitus type 2, with an incomplete direction of research on gestational diabetes as well as an incomplete understanding of its mechanisms, principally because most studies lack large sample sizes. We have offered a fresh insight into the mechanisms underlying insulin resistance seen in GDM, which should be explored though further research.

\section{Acknowledgments}

Funding: Support for this work was provided by the Natural Science Foundation of Inner Mongolia Autonomous Region (2015MS08145).

\section{Footnote}

Reporting Checklist: The authors have completed the MDAR reporting checklist. Available at https://dx.doi. org/10.21037/apm-21-2940

Data Sharing Statement: Available at https://dx.doi. org/10.21037/apm-21-2940

Conflicts of Interest: All authors have completed the ICMJE uniform disclosure form (available at https://dx.doi. org/10.21037/apm-21-2940). Dr. DL reported that support for this work was provided by the Natural Science Foundation of Inner Mongolia Autonomous Region (2015MS08145), and payments were made to Dr. DL. The other authors have no conflicts of interest to declare.

Ethical Statement: The authors are accountable for all aspects of the work in ensuring that questions related to the accuracy or integrity of any part of the work are appropriately investigated and resolved. The study was approved by the Health Office Ethics Commission of the Inner Mongolia Autonomous Region People's Hospital (201811005) and all study subjects signed inform consent documentation. All procedures performed in this study involving human participants were in accordance with the Declaration of Helsinki (as revised in 2013).

Open Access Statement: This is an Open Access article distributed in accordance with the Creative Commons Attribution-NonCommercial-NoDerivs 4.0 International License (CC BY-NC-ND 4.0), which permits the noncommercial replication and distribution of the article with the strict proviso that no changes or edits are made and the original work is properly cited (including links to both the formal publication through the relevant DOI and the license). See: https://creativecommons.org/licenses/by-nc-nd/4.0/.

\section{References}

1. Powe CE, Allard C, Battista MC, et al. Heterogeneous contribution of insulin sensitivity and secretion defects to gestational diabetes mellitus. Diabetes Care 2016;39:1052-5.

2. Garrison A. Screening, diagnosis, and management of gestational diabetes mellitus. Am Fam Physician 2015;91:460-7.

3. Catalano PM, Tyzbir ED, Roman NM, et al. Longitudinal changes in insulin release and insulin resistance in nonobese pregnant women. Am J Obstet Gynecol 1991;165:1667-72.

4. Zhuo C, Jiang R, Lin X, et al. LncRNA H19 inhibits autophagy by epigenetically silencing of DIRAS 3 in diabetic cardiomyopathy. Oncotarget 2017;8:1429-37.

5. Crusell MKW, Hansen TH, Nielsen T, et al. Gestational diabetes is associated with change in the gut microbiota composition in third trimester of pregnancy and postpartum. Microbiome 2018;6:89.

6. Matera AG, Terns RM, Terns MP. Non-coding RNAs: lessons from the small nuclear and small nucleolar RNAs. Nat Rev Mol Cell Biol 2007;8:209-20.

7. Chu C, Spitale RC, Chang HY. Technologies to probe functions and mechanisms of long noncoding RNAs. Nat Struct Mol Biol 2015;22:29-35.

8. Black $M H, W u J$, Takayanagi $M$, et al. Variation in PPARG is associated with longitudinal change in insulin resistance in Mexican Americans at risk for type 2 diabetes. J Clin Endocrinol Metab 2015;100:1187-95.

9. Fan M, Li W, Wang L, et al. Association of SLC30A8 gene polymorphism with type 2 diabetes, evidence from 46 studies: a meta-analysis. Endocrine 2016;53:381-94.

10. Mormile R. Induction of GLUT4 by inhibiting IFN- $\gamma$ : a winning move to halt type 2 diabetes? Int J Colorectal Dis 2016;31:1387.

11. Takamoto I, Kubota N, Nakaya K, et al. TCF7L2 in mouse pancreatic beta cells plays a crucial role in glucose homeostasis by regulating beta cell mass. Diabetologia 2014;57:542-53.

12. Gui W, Zhu WF, Zhu Y, et al. LncRNAH19 improves insulin resistance in skeletal muscle by regulating heterogeneous nuclear ribonucleoprotein A1. Cell 
Commun Signal 2020;18:173.

13. Li Y, Li D, Cheng X. The association between expression of lncRNAs in patients with GDM. Endocr Connect 2021;10:1080-90.

14. Matli B, Schulz A, Koeck T, et al. Distribution of HOMAIR in a population-based cohort and proposal for reference intervals. Clin Chem Lab Med 2021;59:1844-51.

15. Idris I. News and Views September 2019: American Diabetes Association (ADA) 79th scientific session Highlights. Diabetes Obes Metab 2019;21:2183-6.

16. Thompson HR, Madsen KA. The report card on BMI report cards. Curr Obes Rep 2017;6:163-7.

17. Horseman ND, Gregerson KA. Prolactin actions. J Mol Endocrinol 2014;52:R95-106.

18. Di Cianni G, Miccoli R, Volpe L, Lencioni C, et al. Intermediate metabolism in normal pregnancy and in gestational diabetes. Diabetes Metab Res Rev 2003;19:259-70.

19. Buchanan TA, Xiang AH, Page KA. Gestational diabetes mellitus: risks and management during and after pregnancy. Nat Rev Endocrinol2012;8:639-49.

20. Steyn A, Crowther NJ, Norris SA, et al. Epigenetic modification of the pentose phosphate pathway and the

Cite this article as: $\mathrm{Li} \mathrm{Y,} \mathrm{Cheng} \mathrm{X,} \mathrm{Li} \mathrm{D.} \mathrm{LncRNA} \mathrm{RPL13p5}$ gene expression promotes insulin resistance in patients with gestational diabetes. Ann Palliat Med 2021;10(10):11024-11034. doi: 10.21037/apm-21-2940
IGF-axis in women with gestational diabetes mellitus. Epigenomics 2019;11:1371-85.

21. Yang S, Ji Q, Chang B, et al. STC2 promotes head and neck squamous cell carcinoma metastasis through modulating the PI3K/AKT/Snail signaling. Oncotarget 2017;8:5976-91.

22. Oxvig C. The role of PAPP-A in the IGF system: location, location, location. J Cell Commun Signal 2015;9:177-87.

23. Conover CA, Oxvig C. PAPP-A: a promising therapeutic target for healthy longevity. Aging Cell 2017;16:205-9.

24. Becker MA, Haluska P Jr, Bale LK, et al. A novel neutralizing antibody targeting pregnancy-associated plasma protein-a inhibits ovarian cancer growth and ascites accumulation in patient mouse tumorgrafts. Mol Cancer Ther 2015;14:973-81.

25. Jepsen MR, Kløverpris S, Mikkelsen JH, et al. Stanniocalcin-2 inhibits mammalian growth by proteolytic inhibition of the insulin-like growth factor axis. J Biol Chem 2015;290:3430-9.

(English Language Editor: B. Draper) 